\title{
Analysis and development prospects of thermoelectric power generation
}

\author{
Mu Wang \\ North China Electric Power University (Baoding), Baoding, 071000, China \\ 2475593950@qq.com
}

Keywords: Thermoelectric power generation; principle, theoretical analysis model; development prospects.

\begin{abstract}
Thermoelectric power generation technology has many advantages, no medium leakage, no wear, no noise, small size, light weight, convenient movement and high reliability. It is also not limited by temperature, so it shows great superiority in the recovery of low grade heat source utilization. This paper discusses the principle of thermoelectric power generation, explores the theoretical analysis model and summarizes the development prospects of thermoelectric power generation.
\end{abstract}

\section{Introduction}

Research on thermoelectric refrigeration technology at home and abroad mainly focus on three aspects: thermoelectric materials, thermoelectric power generation and structure of the hot and cold side heat dissipation enhancement mode. Due to the low thermoelectric conversion efficiency and hige cost in production, thermal power generation technology has long been mainly used in aerospace and military fields, and less in civilian areas. In recent years, with the development of high performance thermoelectric materials with high merit, the application of thermoelectric generation technology in industrial and civil industries has become possible. At the same time, energy and environmental issues have become increasingly prominent which has led scientists to increase the use of thermoelectric power generation and recovery on utilization of low grade heat source.

\section{The basic principles}

\subsection{Heat source}

The heat source is divided into non nuclear and nuclear heat source. The characteristics of non nuclear heat source are easily obtained and no radioactive pollution, suitable for various environments. We can use the waste heat of circulating water of high temperature reactor to generate electricity to drive the instrument; use thermoelectric devices to turn auto generator exhaust waste heat into electricity; use the temperature difference between the skin and the environment to supply power for watches and use boiler waste heat to generate power.

\subsection{The semiconductor thermoelectric device}

Thermoelectric devices based on the Seebeck effect of semiconductor thermoelectric materials can convert heat directly into electricity devices. The Seebeck effect is a thermal energy conversion to electricity phenomenon. As shown in Figure 1, two different conductors constitute the loop AB. When the two node temperatures $T_{h}, T_{c}$ are different, there is an electric potential loop. This effect is also known as the Seebeck effect and its thermoelectric properties can be described by the Seekbeck coefficient.

If there is a temperature difference $\Delta T=T_{1}-T_{2}$, there will be anelectromotive force in the open position between the conductor $\mathrm{A} X$ and $\mathrm{Y}$, its value is:

$$
\Delta U_{x y}=\alpha_{a b}\left(T_{1}-T_{2}\right)
$$

When the joint temperature difference $\Delta T=T_{1}-T_{2}$ is not large, the relationship is linear, so it can be regarded as a constant, the constant is defined as the Seebeck coefficients of two kinds of conductor. 


$$
\alpha_{a b}=\lim _{\Delta T \rightarrow 0} \frac{\Delta U_{x y}}{\Delta T}=\frac{d U_{x y}}{d T}
$$

Two kinds of materials are usually made for thermoelectric generation module respectively using $\mathrm{P}$ type and $\mathrm{N}$ type semiconductor. $\alpha_{P}, \alpha_{N}$ represent the absolute Seebeck coefficient of $\mathrm{P}$ type semiconductor and $\mathrm{N}$ type semiconductor materials. $\alpha_{P N}$ represents relative Seebeck coefficient. The relationship between $\alpha_{P} 、 \alpha_{N}$ and $\alpha_{P N}$ is

$$
\alpha_{P N}=\alpha_{P}+\left|\alpha_{N}\right|
$$

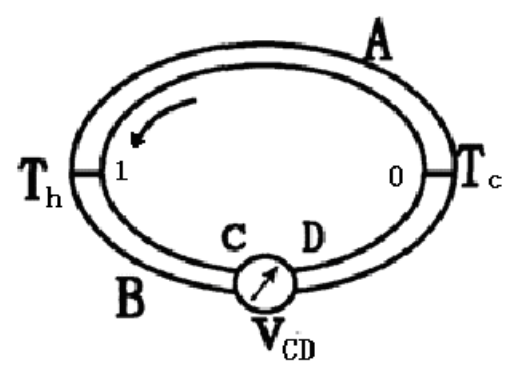

Figure 1 Schematic diagram of the Seebeck effect

The relationship represents that $\mathrm{P}$ type and $\mathrm{N}$ type material monomer thermoelectromotive force total can be the sum of the absolute value of the thermoelectromotive force of these two parts. The microscopic essence of Beck effect can be explained by the change of carrier distribution in the conductor under temperature gradient. For the isolated conductor whose end has not established temperature, the carrier is in uniform distribution in conductor. Once the temperature gradient is established in the conductor, heat carrier has great momentum, tends to spread to the cold end and accumulate in the cold end, making the carrier concentration of the cold end higher than that of the hot end. The accumulation of this charge will enable the destruction of the neutral conductor. On the other hand, the accumulation of charge at the cold end leads to the formation of a self built electric field in the conductor, in order to prevent the further spread of the hot end to the cold end of the carrier. When the conductor reaches equilibrium, there will be no directional movement of net charge in the conductor; the potential formed in both ends in the conductor is called Seebeck potential.

\subsection{Insulation layer}

Adiabatic insulation layer will directly affect the temperature of the hot working temperature of thermoelectric devices, and not influence the semiconductor thermoelectric device and the conversion efficiency of the semiconductor thermoelectric. Insulation layer is mainly composed of thermal insulation materials and a thermal insulation material is the material or composite material significantly resistant to heat.

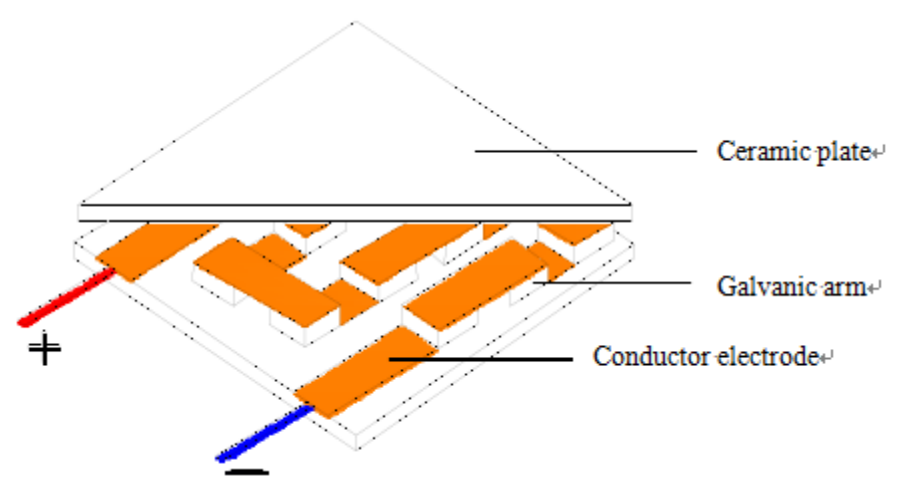

Figure2 Schematic diagram of thermoelectric module 


\subsection{Heat dissipation and strength components}

The heat radiating component is required to produce heat emitted into the environment timely, in order to maintain the temperature difference between the heat source and the thermoelectric cold end. The electrical energy produced by the generator can derive through the power output device. Cavity is usually filled with argon and other protective gas for the protection of the heat source and the thermoelectric device, thermoelectric module schematic diagram shown in Figure 2.

\section{Theoretical analysis model}

The basic thermoelectric power generation unit contains only a pair of $\mathrm{P}$ type and $\mathrm{N}$ type semiconductor thermocouple, being regarded as the ideal model. We can deduce the main performance parameters formula of rational model.

$T_{h}$ is high temperature heat source temperature;

$T_{c}$ is environment temperature;

$T_{1}$ is semiconductor thermoelectric generator hot end temperature;

$T_{2}$ is semiconductor thermoelectric generator cold end temperature;

$Q_{h}$ and $Q_{c}$ are the heat flux flow to and out of heat electric appliance per unit time;

$U_{h}$ is the heat conduction rate of hot end heat exchanger;

$U_{c}$ is the heat conduction rate of cold end heat exchanger;

$A_{h}$ and $A_{c}$ are the heat transfer area of hot end and cold end;

$R_{l}$ is the load resistance heat generator. The generator consists of $\mathrm{N}$ type and $\mathrm{P}$ type of semiconductor element in series with the load resistance. Assume Seebeck coefficient with $\mathrm{N}$ type and $\mathrm{P}$ type semiconductor element is $\alpha_{n}$ and $\alpha_{p}$, resistivity is $\rho_{n}$ and $\rho_{p}$, thermal conductivity is $k_{n}$ and $k_{p}$. We also assume that the semiconductor element side is in insulation isolation and the Joule heat is the average distribution of galvanic arm ends. When the electrical is stable, under the boundary conditions, the heat conduction equation can be obtained as follows

$$
\begin{aligned}
& Q_{h}=\alpha I T_{1}-\frac{1}{2} I^{2} R+K\left(T_{1}-T_{2}\right) \\
& Q_{c}=\alpha I T_{2}-\frac{1}{2} I^{2} R+K\left(T_{1}-T_{2}\right)
\end{aligned}
$$

\section{Conclusions}

Thermoelectric power generation technology has been widely used in waste heat recovery and low temperature heat source power and other fields. Compared with the traditional heat recovery energysaving technology, thermoelectric power generation technology will be an effective supplement. With the deepening of thermoelectric materials and the improvement of the performance, it is believed that heat will be used more and more. It is of great significance when China is in the transition from a energy consumption state transition to a resource-saving and environment-friendly society.

\section{References}

[1]Deng Changsheng, the Principle and Application of Solar Energy, Chemical Industry Press, 2010, 35(in Chinese)

[2]Jia Lei, Chen Zeshao, Research on thermal conductivity of thermoelectric generator, Acta Energies Solaris Sinica, 2004, 25(6):816-819

[3]Yang Yongjie, Zhang Yusheng, Design of a PM 2.5 detecting sensor, Transducer and microsystem, 2014, 33(3).71-78 